Research Paper

\title{
Independent Prognostic Value of Hypoxia-inducible Factor 1-alpha Expression in Small Cell Lung Cancer
}

\author{
Chang-Sheng Lin ${ }^{1,2}$, Tu-Chen Liu ${ }^{3}$, Ming-Tsung Lee ${ }^{4}$, Shun-Fa Yang ${ }^{1,5,}$, , Thomas Chang-Yao Tsao ${ }^{6,7,}, \bowtie$ \\ 1. Institute of Medicine, Chung Shan Medical University, Taichung, Taiwan \\ 2. Department of Chest Medicine, Show Chwan Memorial Hospital, Changhua, Taiwan \\ 3. Department of Chest Medicine, Cheng-Ching General Hospital, Taichung, Taiwan \\ 4. Research Assistant Center, Chang Hua Show Chwan Health Care System, Changhua, Taiwan \\ 5. Department of Medical Research, Chung Shan Medical University Hospital, Taichung, Taiwan \\ 6. Division of Chest, Department of Internal Medicine, Chung Shan Medical University Hospital, Taichung, Taiwan \\ 7. School of Medicine, Chung Shan Medical University, Taichung, Taiwan
}

$\square$ Corresponding author: Thomas Chang-Yao Tsao, M.D., Ph.D. Division of Thoracic Medicine, Chung Shan University Hospital and Chung Shan Medical University, Taichung, Taiwan. Tel: +886-4-24730022 ext. 11020. Fax: +886-4-24759950. E-mail: his885889@gmail.com Or Shun-Fa Yang, PhD. Institute of Medicine, Chung Shan Medical University, Taichung, Taiwan. E-mail: ysf@csmu.edu.tw

(C) Ivyspring International Publisher. This is an open access article distributed under the terms of the Creative Commons Attribution (CC BY-NC) license (https://creativecommons.org/licenses/by-nc/4.0/). See http://ivyspring.com/terms for full terms and conditions.

Received: 2017.02.06; Accepted: 2017.05.17; Published: 2017.07.18

\begin{abstract}
Hypoxia is an important factor in tumor angiogenesis, metastasis, and resistance to chemotherapy or radiotherapy, and may be an indicator of poor prognosis. The transcription factor hypoxia-inducible factor 1 (HIF-l) is the key regulator of the hypoxic state. This study was designed to evaluate the prognostic value of HIF-l $\alpha$ expression in small cell lung cancer (SCLC). Forty-three paraffin-embedded biopsy materials were examined using immunohistochemistry. Our results indicated that the expression of HIF-l $\alpha$ was high in males, and patients with poor Eastern Cooperative Oncology Group (ECOG) performance status and metastases. To elucidate the prognostic value of HIF-1 $\alpha$ expression, Kaplan-Meier analysis was carried out and the results showed that patients with high HIF-1 $\alpha$ expression had a poorer prognosis than patients with low expression of HIF-1 $\alpha$. After adjusting clinical parameters by the Cox proportional hazards model, our results demonstrated that high HIF-l $\alpha$ expression is an independent prognostic factor for SCLC with a 39.2-fold risk of death $(p<0.003)$. In conclusion, we have provided evidence that HIF- $1 \alpha$ expression has significant value in predicting survival of patients with SCLC and is an independent prognostic factor beyond ECOG performance and metastasis status.
\end{abstract}

Key words: small cell lung cancer, hypoxia-inducible factor-1 alpha, immunohistochemistry, survival

\section{Introduction}

Lung cancer is the leading cause of cancer-related death worldwide. Human lung cancers are classified into small cell lung cancer (SCLC) and non-small cell lung cancer (NSCLC) groups, the latter consisting of adenocarcinoma, squamous cell carcinoma, bronchioalveolar carcinoma, and large-cell carcinoma. Despite advances in lung cancer therapy, the average 5-year survival rate is only $18 \%$ [1]. Between $15 \%$ and $25 \%$ of all lung cancer cases are classified histologically as SCLC, which is characterized by rapid growth and a high metastatic potential [2]. The natural history of SCLC reveals earlier dissemination and higher mortality than that of NSCLC. In general, SCLC is considered to be a systemic disease, even if SCLC appears to be confined to the chest at the time of diagnosis. Patients with SCLC have decreased longevity and are rarely cured with the currently available therapies.

According to the two-stage system of the Veterans Administration Lung Cancer Group (VALG), SCLC is classified as extensive (ED-SCLC) and limited disease (LD-SCLC). The pre-treatment prognostic factors that consistently predict prolonged survival include good performance status, female 
gender, and LD-SCLC. The median survival for untreated LD-SCLC and ED-SCLC patients is 4-6 months and 5-9 weeks, respectively. Of note, the survival of SCLC patients could be improved significantly with effective therapy [3].

Hypoxia is a critical factor that impacts cell proliferation, survival, angiogenesis, immunosurveillance, metabolism, tumor invasion, and metastasis, and is associated with a worse prognosis for cancer patients [4-7]. Transcription factor hypoxia-inducible factor 1 (HIF-1) is the key regulator responsible for the induction of genes that facilitate adaptation and survival of cells, and the entireorganism, from normoxia $\left(\sim 21 \% \mathrm{O}_{2}\right)$ to hypoxia $\left(\sim 1 \% \mathrm{O}_{2}\right)[8,9]$. There are two main characteristics of solid tumors (neovascularization and increased glycolysis), which represent adaptations to a hypoxic microenvironment and are correlated with tumor invasion, metastasis, and lethality. HIF-1a overexpression has been previously reported to be associated with a poor prognosis in breast, oropharyngeal, and cervical cancers [10-13]. Moreover, the correlation between the presentation of HIF-1a and the prognosis of NSCLC has been analyzed [14-16]. Specifically, Giatromanolaki showed that HIF-1a is associated with VEGF expression, but is not significantly correlated with NSCLC prognosis [14], and Swinson had revealed that HIF-1a expression is associated with a poor prognosis [15]. Moreover, a systematic review also reports that HIF-1a might serve as important factors in evaluating prognosis of lung cancer [16]. There are no data, however, showing an association between HIF-1a expression and SCLC prognosis. In the current study, we examined HIF-1a protein expression in 43 SCLC specimens using immunohistochemistry and evaluated the role of HIF-1a in influencing the prognosis of SCLC.

\section{Materials and methods}

\section{Patient and study design}

The original biopsy materials were obtained from bronchoscopic or computer tomography-guided needle specimens. Archival paraffin-embedded biopsy materials from 43 SCLC (all patients received at least 2 courses of chemotherapy with cisplatin [25 $\mathrm{mg} / \mathrm{m}^{2} /$ day] and etoposide $\left[100 \mathrm{mg} / \mathrm{m}^{2} /\right.$ day] for 3 days monthly) were retrieved and $4-\mu \mathrm{m}$ tissue sections were cut on slides. Histologic diagnoses were made using hematoxylin and eosin (H\&E) staining. The Institutional Review Board of Show Chwan Memorial Hospital approved the study proposal (IRB No. 1000909).

\section{Immunohistochemistry for HIF-1 $\alpha$}

Slide sections were dewaxed with xylene and rehydrated through a gradient concentration of alcohol. After the sections were microwaved, the primary monoclonal HIF-1a antibody (SC-53546, dilution 1:50; Santa Cruz Biotechnology, Inc., Santa Cruz, CA, USA) was applied as described previously [17]. The secondary antibody was applied at room temperature for $15 \mathrm{~min}$ after twice-washing in water and Tris buffer for $5 \mathrm{~min}$. Then, the slide was washed again using the abovementioned procedures. The slides were then incubated with avidin-HRP for 30 min and washed again. The color was developed with 3, 3'-diaminobenzidine solution (DAB) for $5 \mathrm{~min}$. Gill's hematoxylin was used for counterstaining and the staining time was $<1 \mathrm{~min}$. The stained slide was immersed in 95\% alcohol, then in absolute alcohol for $5 \mathrm{~min}$. Negative controls were not exposed to primary antibody. The stained slides were cover-slipped and observed under light microscopy at an optical power of $\times 40$.

\section{Scoring of HIF- $1 \alpha$ expression}

The immunohistochemical results for HIF-1a protein were examined and the scored results were assessed by three board-certified pathologists. Every slide was given a score according to the intensity of the nuclear staining, as follows: no staining or nuclear staining in $<1 \%$ of the cells, negative $(-)$; nuclear staining in $1 \%-10 \%$ of cells, mild $(+)$; nuclear staining in $10 \%-50 \%$ of cells, moderate $(++)$; and nuclear staining in $>50 \%$ of cells, strong $(+++)$. In the current study, moderate $(++)$ and strong scores $(+++)$ were considered to be high immunostaining and negative $(-)$ and mild scores $(+)$ were considered to be low immunostaining.

\section{Statistics}

Fisher's exact or chi-square test was applied for statistical analysis. Survival curves were plotted using the Kaplan-Meier method and statistical significance was assessed using the log-rank test. Univariate analysis was analyzed by the Cox proportional hazards regression model and the statistical analysis was performed using SPSS for Windows (version 12; SPSS, Inc., Chicago, IL, USA). AP $<0.05$ was considered to be statistically significant.

\section{Results}

\section{Patient Characteristics}

Forty-three SCLC patients were included in this retrospective study (Table 1 ). The median age of the patients was $70 \pm 9.82$ years (range, $47-87$ years), and male patients were predominant $(95.3 \%)$. Of the 
(a)

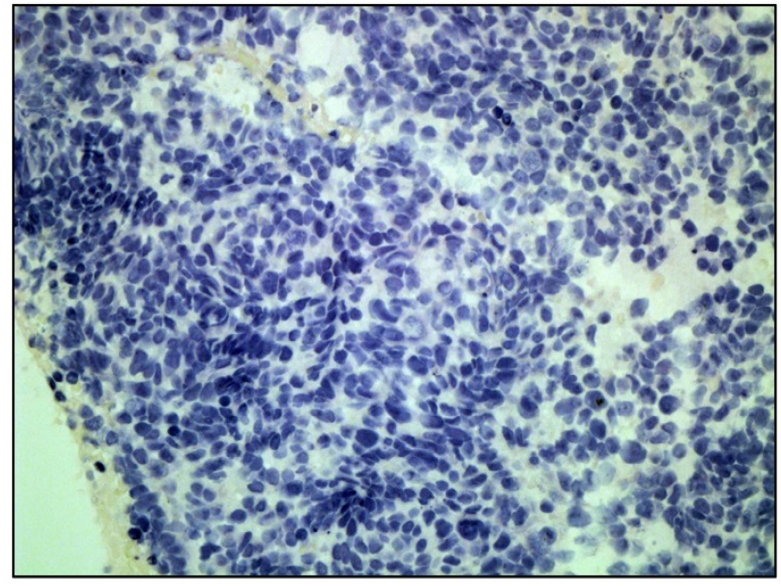

(b)

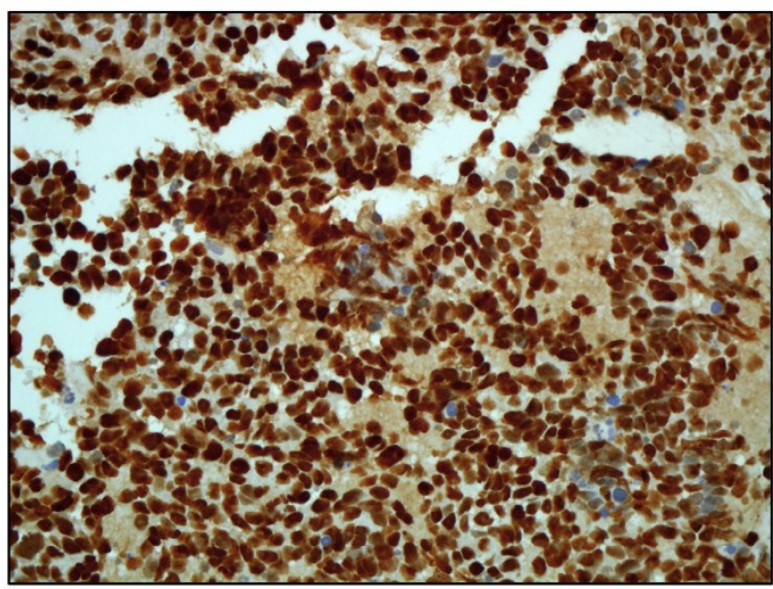

Figure 1. Representative of HIF-1 $\alpha$ protein immunostaining in paraffin sections of SCLC tumors. (a). low immunostaining (40X). (b). high. immunostaining (40X).

patients, 93\% were smokers or ever-smokers. The Eastern Cooperative Oncology Group (ECOG) performance status score of all patients was $\leq 3$. As expected, a high distant metastases rate was common at the time of diagnosis of SCLC (83.7\%).

Table 1. Relationships between clinical parameters and HIF-1 $\alpha$ expression in 43 SCLC patients

\begin{tabular}{lllll}
\hline Characteristics & $\begin{array}{l}\text { No. of } \\
\text { cases }\end{array}$ & $\begin{array}{l}\text { Low }(\%) \\
\mathrm{n}=17\end{array}$ & $\begin{array}{l}\text { High }(\%) \\
\mathrm{n}=26\end{array}$ & $\mathrm{p}^{2}$ \\
\hline Age (years) ${ }^{1}$ (range) & $\begin{array}{l}70 \pm 9.82 \\
(47-87)\end{array}$ & & & \\
& 18 & $7(39)$ & $11(61)$ & 0.941 \\
$<70$ & 25 & $10(40)$ & $15(60)$ & \\
$\geqq 70$ & & & & \\
Gender & 2 & $2(100)$ & $0(0)$ & 0.0493 \\
Female & 41 & $15(37)$ & $26(63)$ & \\
Male & & & & \\
$\begin{array}{l}\text { ECGO performance } \\
\text { status }\end{array}$ & 3 & $3(100)$ & $0(0)$ & $<0.001$ \\
0 & 13 & $12(92)$ & $1(8)$ & \\
1 & 16 & $2(13)$ & $14(87)$ & \\
2 & 11 & $0(0)$ & $11(63)$ & \\
3 & & & & \\
Smoking status & 3 & $1(33)$ & $2(67)$ & $1.000^{3}$ \\
$\begin{array}{l}\text { Never smoked } \\
\text { Current smoker or ever }\end{array}$ & 40 & $16(40)$ & $24(60)$ & \\
smoked & & & & \\
$\begin{array}{l}\text { Distant metastasis } \\
\text { Negative }\end{array}$ & 7 & $7(100)$ & $0(0)$ & $0.001^{3}$ \\
Positive & 36 & $10(28)$ & $26(72)$ & \\
\hline
\end{tabular}

1. Mean \pm SD

2. Chi-square test for categorical variables

${ }^{3}$. Fisher's exact test

\section{HIF-1 $\alpha$ expression scores}

Representative immunohistostaining results of HIF-1a are shown in Figure 1. Low and high HIF-1a immunostaining expression is shown in Figure 1 (a) and (b), respectively. Of the 43 cases analyzed, the levels of HIF-1a expression were as follows: 7 cases $(16.3 \%)$ with negative staining (-); 10 cases $(23.3 \%)$ with mild staining $(+)$; 9 cases $(20.9 \%)$ with moderate staining $(++)$; and 17 cases $(39.5 \%)$ with strong staining $(+++)$. In the current study, 26 cases $(60.4 \%)$ had high expression of HIF-1a.

\section{Relationships between HIF- $1 \alpha$ expression and clinical pathologic parameters in SCLC patients}

Among the studied clinic-pathologic parameters, including age, gender, ECOG performance status, smoking status, and distant metastases, statistically significant associations between HIF-1a expression and gender, smoking status, ECOG performance status, and distant metastases were observed, as shown in Table 1 ( $p=0.049$ for gender; $p<0.001$ for ECOG performance status; and $\mathrm{p}=0.001$ for distant metastases). There were two female patients in the study with low HIF-1a immunostaining. Interestingly, HIF-1a expression was concordant with the ECOG performance status $(\mathrm{p}<0.001)$. Patients with a poor ECOG performance status had high HIF-1a expression. Furthermore, HIF-1a was expressed in 0 and $72 \%$ of distant metastasis-negative and -positive patients, respectively. Our results suggest that ECOG performance status and distant metastases are highly corrected with HIF-1a expression.

\section{Prognostic value of HIF- $1 \alpha$ expression in SCLC patients}

The median survival of all patients was $167 \pm$ 14.42 days ( $95 \% \mathrm{CI}, 138.72$ - 195.27 days). According to the results of immunohistostaining, the median survival of the low- and high-staining groups was 311 \pm 12.35 days ( $95 \%$ CI, 286.8 - 335.2 days) and $102 \pm 15.3$ days (95\% CI, 72.02 - 131.92 days), respectively. The association of HIF-1a expression with various clinic-pathologic parameters with patient survival 
was determined by univariate analysis. As expected and as shown in Table 2, poor ECOG performance status and distant metastasis-positive patients had shorter survival than good ECOG performance status and distant metastasis-negative patients $(\mathrm{p}<0.001$ for ECOG performance status; and $\mathrm{p}<0.001$ for distant metastasis). The results showed that patients with high HIF-1a expression had significantly shorter survival than patients with low HIF-1a expression (Table 2). Figure 2 shows that patients who express HIF-1a had significantly shorter overall survival based on Kaplan-Meier survival curves (log-rank, $\mathrm{p}<0.001)$. Moreover, Cox regression analysis data indicated the patients with high HIF-1a expression had a significantly worse overall survival than patients with low HIF-1a expression ( $\mathrm{p}=0.003$, Table 3 ). Among the study cases, the risk ratio (RR) of age, ECOG performance status, and distant metastases were 2.372-, 4.286-, and 11.858-fold, respectively $(\mathrm{p}=0.025$ and $95 \% \mathrm{CI}, 1.114-5.049$ for age; $\mathrm{p}=4.286$ and 95\% CI, 0.726-25.303 for ECOG performance status; $\mathrm{p}=0.003$ and $95 \% \mathrm{CI}, 2.259-62.243$ for distant metastases). Interestingly, the RR of patients with high HIF-1a expression was 39.207-fold patients with low HIF-1a expression. The RR of HIF-1a expression (39.207-fold) was higher than the RR of distant metastases (11.858-fold). Thus, HIF-1a expression is a more effective independent prognostic factor than stage status in patients with SCLC.

Table 2. Univariate analysis of influences of clinical characteristics on overall survival duration of SCLC patients

\begin{tabular}{|c|c|c|c|}
\hline Characteristics & $\begin{array}{l}\text { No. of } \\
\text { cases }\end{array}$ & $\begin{array}{l}\text { Median survival } \\
\text { (days) }\end{array}$ & Log-rank ${ }^{1}$ \\
\hline \multicolumn{4}{|l|}{ Age (years) } \\
\hline$<70$ & 18 & 183 & \multirow[t]{2}{*}{0.3646} \\
\hline$\geqq 70$ & 25 & 167 & \\
\hline \multicolumn{4}{|l|}{ Gender } \\
\hline Female & 2 & 288 & \multirow[t]{2}{*}{0.666} \\
\hline Male & 41 & 167 & \\
\hline \multicolumn{4}{|l|}{ ECGO performance status } \\
\hline 0 & 3 & 537 & \multirow[t]{4}{*}{$<0.001$} \\
\hline 1 & 13 & 308 & \\
\hline 2 & 16 & 161 & \\
\hline 3 & 11 & 61 & \\
\hline \multicolumn{4}{|l|}{ Smoking status } \\
\hline Never smoked & 3 & 181 & \multirow[t]{2}{*}{0.614} \\
\hline $\begin{array}{l}\text { Current smoker or ever } \\
\text { smoked }\end{array}$ & 40 & 167 & \\
\hline \multicolumn{4}{|l|}{ Distant metastasis } \\
\hline Negative & 7 & 386 & \multirow[t]{2}{*}{$<0.001$} \\
\hline Positive & 36 & 156 & \\
\hline \multicolumn{4}{|l|}{ HIF-1a immunostaining } \\
\hline Low & 17 & 311 & \multirow[t]{2}{*}{$<0.001$} \\
\hline High & 26 & 102 & \\
\hline
\end{tabular}

1. Log-rank p-values for categorical variables were statistically analyzed by Kaplan-Meier test.
Table 3. Cox regression analysis of various potential prognostic factors in SCLC patients 1

\begin{tabular}{|c|c|c|c|c|}
\hline Variables & RR & Unfavorable/Favorable & $\mathrm{p}$ & $95 \% \mathrm{CI}$ \\
\hline $\begin{array}{l}\text { HIF-1a } \\
\text { immunostaining }\end{array}$ & 39.207 & high/low & 0.003 & $3.355-458.219$ \\
\hline Age & 2.372 & $\geqq 70 /<70$ & 0.025 & 1.114-5.049 \\
\hline Gender & 0.824 & male/female & 0.868 & $0.084-8.080$ \\
\hline $\begin{array}{l}\text { ECGO } \\
\text { performance status }\end{array}$ & 4.286 & $3,4 / 1,2$ & 0.108 & $0.726-25.303$ \\
\hline Smoking status & 1.110 & $\begin{array}{l}\text { current or ever } \\
\text { smoked/never smoked }\end{array}$ & 0.874 & $=0.305-4.037$ \\
\hline Distant metastasis & 11.858 & positive/negative & 0.003 & $2.259-62.243$ \\
\hline
\end{tabular}

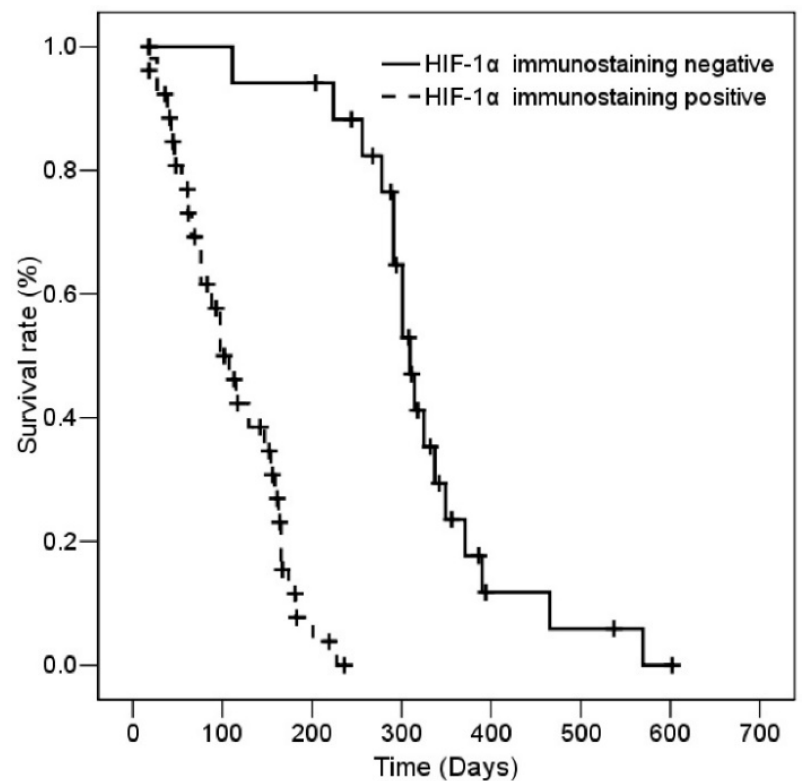

Figure 2. Kaplan-Meier survival of 43 SCLC patients with high and low HIF-1 $\alpha$ immunostaining.

\section{Discussion}

HIF-1a is an important regulator in tumor angiogenesis and distant metastases. HIF-1a is overexpressed in many types of human cancers and is associated with a poor prognosis. HIF-1a expression was demonstrated in $84 \%$ of the patients with SCLC in the current study. Also, patients with a higher level of HIF-1a expression had significantly shorter survival times and more distant metastases than patients with a low level of HIF-1a expression.

HIF-1a expression has been reported to be increased in 13 types of cancer, including lung, prostate, breast, and colon carcinomas, which are the leading causes of cancer mortality in the US [18]. HIF-1a overexpression was demonstrated in $55.8 \%$ of patients with NSCLC in one study, and HIF-1a protein was overexpressed in $66.7 \%(6 / 9)$ of patients with SCLC in another study $[19,20]$. In the current 
study, HIF-1a was detected in $83.7 \%(36 / 43)$ of patients with SCLC, $60.4 \%(26 / 34)$ of whom had high expression. HIF-1a expression is more prominent in patients with SCLC than NSCLC, which is consistent with clinical findings. This observation might explain why SCLC is more malignant and metastasizes earlier.

The prognostic significance of HIF-1a expression has been evaluated in several solid tumors. Increased HIF-1a expression has been reported to be associated with a poor prognosis in lymph node-positive breast cancer and non-metastatic oropharyngeal cancer [10, 11]. Two studies evaluated the relationship between the presentation of HIF-1a and the prognosis in patients with NSCLC. One study showed that HIF-1a protein is associated with expression of vascular endothelial growth factor (VEGF), platelet-derived endothelial cell growth factor, and basic fibroblast growth factor in patients with NSCLC, but was not significantly associated with prognosis [14]. The other study reported that higher HIF-1a expression is associated with a poorer prognosis [15]. Low HIF-1a expression in ED-SCLC patients who were treated with frontline platinum-based chemotherapy had better overall survival rate [19]. Luan reported that HIF-1a and HIF-2a expression is related to shorter overall survival, which was similar to our results; however, only HIF-2a expression has been recommended to be an independent prognostic marker [20].

Inhibition of HIF-1a activity has become an effective anti-tumor therapy for various tumors [21]. Moreover, Bryant et al. described that targeting hypoxia maybe important in the development of novel therapies for SCLC [22]. Cisplatin and etoposide are usually used as first-line chemotherapy for SCLC, and can significantly prolong survival; however, most SCLC patients treated with first-line chemotherapy will eventually relapse. Topotecan is commonly used as second-line chemotherapy for SCLC. In an in vitro study, topotecan reduced the hypoxic up-regulation of HIF-1a, and reduced the HIF-1 transcriptional response to hypoxia by inhibiting the HIF-1 transcriptional activation pathway through inhibition of HIF-1a translation [23]. Lund reported that patients with very hypoxic tumors might benefit, in particular from topotecan treatment, and the anti-HIF effect of topotecan should be taken into consideration in these patients [24]. Our study presented a wide range of survival time (18-602 days) among the 43 SCLC patients. All of our patients received cisplatin and etoposide as first-line chemotherapy. Therefore, topotecan might be considered as first-line chemotherapy in SCLC patients with high HIF-1a expression to achieve better survival.
In summary, we showed HIF-1a expression in most patients with SCLC. In addition, patients with a higher level of HIF-1a expression had significantly shorter survival times and more distant metastases in the current study. Expression of HIF-1a could be a more effective independent prognosis factor than ECOG performance and distant metastasis in SCLC patients. Based on our data, we recommend that SCLC patients with high HIF-1a expression be treated with a drug that inhibits HIF-1a (topotecan), which we predict will lead to better survival.

\section{Acknowledgments}

This study was supported by the grant from Chang Shan Medical University and Hospital (CSH-2011-C014).

\section{Competing Interests}

The authors have declared that no competing interests exist.

\section{References}

[1] Siegel RL, Miller KD and Jemal A. Cancer statistics, 2016. CA Cancer J Clin 2016; 66: 7-30.

[2] Howe HL, Wingo PA, Thun MJ, et al. Annual report to the nation on the status of cancer (1973 through 1998), featuring cancers with recent increasing trends. J Natl Cancer Inst 2001; 93: 824-842.

[3] Zhang $\mathrm{X}, \mathrm{Yu} \mathrm{J}$, Zhu H, et al. Consolidative thoracic radiotherapy for extensive stage small cell lung cancer. Oncotarget 2017;

[4] Ruan K, Song G, Ouyang G. Role of hypoxia in the hallmarks of human cancer. J Cell Biochem 2009; 107: 1053-1062.

[5] Chen MK, Chiou HL, Su SC, et al. The association between hypoxia inducible factor-1alpha gene polymorphisms and increased susceptibility to oral cancer. Oral Oncol 2009; 45: e222-226.

[6] Hsiao PC, Chen MK, Su SC, et al. Hypoxia inducible factor-1alpha gene polymorphism G1790A and its interaction with tobacco and alcohol consumptions increase susceptibility to hepatocellular carcinoma. J Surg Oncol 2010; 102: 163-169.

[7] Lin CW, Yang WE, Lee WJ, et al. Lipocalin 2 prevents oral cancer metastasis through carbonic anhydrase IX inhibition and is associated with favourable prognosis. Carcinogenesis 2016; 37: 712-722.

[8] Semenza GL. HIF-1 mediates metabolic responses to intratumoral hypoxia and oncogenic mutations. J Clin Invest 2013; 123: 3664-3671.

[9] Semenza GL. Hypoxia-inducible factors: coupling glucose metabolism and redox regulation with induction of the breast cancer stem cell phenotype. EMBO J 2017; 36: 252-259.

[10] Schindl M, Schoppmann SF, Samonigg H, et al. Overexpression of hypoxia-inducible factor 1alpha is associated with an unfavorable prognosis in lymph node-positive breast cancer. Clin Cancer Res 2002; 8: 1831-1837.

[11] Aebersold DM, Burri P, Beer KT, et al. Expression of hypoxia-inducible factor-1alpha: a novel predictive and prognostic parameter in the radiotherapy of oropharyngeal cancer. Cancer Res 2001; 61: 2911-2916.

[12] Peng X, Gong F, Chen Y, et al. Autophagy promotes paclitaxel resistance of cervical cancer cells: involvement of Warburg effect activated hypoxia-induced factor 1-alpha-mediated signaling. Cell Death Dis 2014; 5: e1367.

[13] Martinho O, Silva-Oliveira R, Cury FP, et al. HER Family Receptors are Important Theranostic Biomarkers for Cervical Cancer: Blocking Glucose Metabolism Enhances the Therapeutic Effect of HER Inhibitors. Theranostics 2017; 7: 717-732.

[14] Giatromanolaki A, Koukourakis MI, Sivridis E, et al. Relation of hypoxia inducible factor 1 alpha and 2 alpha in operable non-small cell lung cancer to angiogenic/molecular profile of tumours and survival. Br J Cancer 2001; 85: 881-890.

[15] Swinson DE, Jones JL, Cox G, et al. Hypoxia-inducible factor-1 alpha in non small cell lung cancer: relation to growth factor, protease and apoptosis pathways. Int J Cancer 2004; 111: 43-50.

[16] Ren W, Mi D, Yang K, et al. The expression of hypoxia-inducible factor-1alpha and its clinical significance in lung cancer: a systematic review and meta-analysis. Swiss Med Wkly 2013; 143: w13855.

[17] Lee SS, Tsai CH, Yang SF, Ho YC, Chang YC. Hypoxia inducible factor-1alpha expression in areca quid chewing-associated oral squamous cell carcinomas. Oral Dis 2010; 16: 696-701. 
[18] Zhong H, De Marzo AM, Laughner E, et al. Overexpression of hypoxia-inducible factor 1alpha in common human cancers and their metastases. Cancer Res 1999; 59: 5830-5835.

[19] Lee GW, Go SI, Cho YJ, et al. Hypoxia-inducible factor-1alpha and excision repair cross-complementing 1 in patients with small cell lung cancer who received front-line platinum-based chemotherapy: a retrospective study. J Thorac Oncol 2012; 7: 528-534.

[20] Luan Y, Gao C, Miao Y, et al. Clinicopathological and prognostic significance of HIF-1alpha and HIF-2alpha expression in small cell lung cancer. Pathol Res Pract 2013; 209: 184-189.

[21] Semenza GL. Targeting HIF-1 for cancer therapy. Nat Rev Cancer 2003; 3: 721-732.

[22] Bryant JL, Meredith SL, Williams KJ, White A. Targeting hypoxia in the treatment of small cell lung cancer. Lung Cancer 2014; 86: 126-132.

[23] Rapisarda A, Uranchimeg B, Scudiero DA, et al. Identification of small molecule inhibitors of hypoxia-inducible factor 1 transcriptional activation pathway. Cancer Res 2002; 62: 4316-4324.

[24] Lund EL, Hansen LT, Kristjansen PE. Augmenting tumor sensitivity to topotecan by transient hypoxia. Cancer Chemother Pharmacol 2005; 56: $473-480$ 